\title{
A Psychometric Comparison of Two Carer Quality of Life Questionnaires in Huntington's Disease: Implications for Neurodegenerative Disorders
}

\author{
Peter Hagell ${ }^{\mathrm{a}, *}$ and Stephen Smith ${ }^{\mathrm{b}}$ \\ ${ }^{a}$ The PRO-CARE Group, School of Health and Society, Kristianstad University, Kristianstad, Sweden \\ ${ }^{\mathrm{b}}$ School of Nursing Sciences, Faculty of Medicine and Health, University of East Anglia, Norwich, England, UK
}

\begin{abstract}
.
Background: The carer impact of neurodegenerative disorders such as Huntington's disease (HD) is vast. Attempts to measure carer QoL in neurodegenerative disorders include the three-dimensional (Practical aspects of Caregiving, PC; Satisfaction with Life, SL; Feelings about Living with Huntington's disease, FL) Huntington's Disease Quality of Life Battery for Carers (HDQoL-C) and the unidimensional Alzheimer's Carer's Quality of Life Inventory (ACQLI). However, evidence regarding their psychometric properties is sparse.

Objectives: To test and compare the psychometric properties of the HDQoL-C, its short-form, and the ACQLI among HD carers. Methods: Data from $61 \mathrm{HD}$ carers (36 women; mean age, 55) were analysed using traditional psychometric methods.

Results: Data completeness was good ( $>95 \%$ computable scale scores) but compromised for the PC and total HDQoL-C scales ( $\leq 80 \%$ computable scale scores). Scaling assumptions were supported for the SL and ACQLI scales (corrected item-total correlations $\geq 0.38$; scaling success rates, 94-100\%) but not for the PC, FL or total HDQoL-C scales (corrected item-total correlations $\geq 0.08$; scaling success rates, $39-62 \%$ ). Floor/ceiling effects were $\leq 9.8 \%$. Reliabilities were $\geq 0.84$, except for the PC scale (0.62).

Conclusions: The HDQoL-C failed to exhibit suitability as a HD carer outcome measure, as two of its three scales did not meet basic psychometric criteria. The third scale (SL) did not outperform the ACQLI. This suggests that carer impact is not disease specific across neurodegenerative disorders.
\end{abstract}

Keywords: Alzheimer's disease, caregivers, Huntington's disease, neurodegeneration, quality of life, reliability, validity

\section{INTRODUCTION}

Neurodegenerative disorders such as Huntington's disease (HD), Parkinson's disease (PD), and Alzheimer's disease (AD) negatively impact not only diagnosed individuals but also family caregivers [1-4]. While such conditions have disease specific features

*Correspondence to: Peter Hagell, PhD, School of Health and Society, Kristianstad University, SE-291 88 Kristianstad, Sweden. Tel.: +46 44 204056; Fax: +46 44 204043; E-mail: Peter.Hagell@ hkr.se. there are also cross-diagnostic similarities, particularly from a real-life day-to-day and family carer perspective [5]. For instance, these and related conditions are all associated with varying degrees and types of impaired mobility, movement disorders, cognitive impairments, behavioural and psychological changes [5, 6]. It therefore appears reasonable to expect similarities in their carer impact.

Indeed, caregiver burden has been found similar between carers of people with dementia, stroke and PD [7], and caregiver needs have been found common 
between PD and AD carers [8]. Similarly, the perceived content validity of a quality of life (QoL) questionnaire for $\mathrm{AD}$ carers has been supported both among $\mathrm{AD}$ and PD carers [9]. However, it has been argued that carers of people with HD experience a unique burden [10]. Heritability of the disease, often necessitating caring for multiple individuals, earlier onset with longer duration of the caregiving role and greater likelihood of caregivers being younger compared to conditions such as $\mathrm{AD}$ have been suggested to make HD caregiving particularly difficult [11].

The importance of taking caregiver perspectives into account has been increasingly recognized over the past decade or so $[1-5,7,8,10,11]$. This is also reflected in the proposal of a number of QoL scales targeting caregivers of people with neurodegenerative disorders [10, 12-15]. Such tools may enable understanding regarding the impact of disease and efficacy of interventions from a wider perspective than that of the clinician or the patient. A prerequisite, however, is that such scales are theoretically well-defined, coupled with supporting evidence regarding their psychometric (measurement) properties [16, 17].

Here we evaluate two caregiver QoL scales among carers of people with HD. The study had two main objectives. First, to test and compare the psychometric properties of the Huntington's Disease Quality of Life Battery for Carers (HDQoL-C) [10] and the Alzheimer's Carer's Quality of Life Inventory (ACQLI) [12]. Specifically, we investigated data completeness, scaling assumptions, internal construct validity, targeting, and reliability using traditional test theory methodology. A secondary objective was to explore whether a questionnaire developed for carers of people with one neurodegenerative disorder (i.e., the ACQLI for AD carers) can be useful among carers of people with another neurodegenerative disorder (i.e., HD). If so, this would provide additional evidence for similarities of carer impact across neurodegenerative disorders, and support the feasibility of a common neurodegenerative carer QoL measure.

\section{MATERIALS AND METHODS}

\section{Sample}

Data were taken from a survey of 61 people (36 women) listed as the primary carer of a person with $\mathrm{HD}$ at a UK neurology clinic. The study was approved by the local research ethics committee. Following a positive response to an invitation letter, all participants provided written informed consent and were then provided with the questionnaires. All participants completed the survey independently, but eight respondents did so in the presence of an investigator (SS). Participants' mean age was $55.2(\mathrm{SD}, 12.1)$ years, $51(84 \%)$ were married/living as married, and $15(26 \%)$ had a university degree. They had been caring for a person with HD for a mean of $6.3(\mathrm{SD}, 7.6)$ years and the majority (53\%) spent more than 30 hours a week caring.

\section{Questionnaires}

The HDQoL-C was developed for HD carers [10, 18] and consists of 34 items (Table 1) proposed to represent three domains (scales): Practical aspects of Caregiving (PC; 9 items), Satisfaction with Life (SL; 8 items) and Feelings about Living with HD (FL; 17 items). It was developed based on a generic 7-domain QoL instrument that was modified by adding, revising and removing items; content validity was assessed by two QoL experts and two HD experts [10]. Items are scored using an 11-grade (0-10) numerical rating scale, summed and transformed into a 0-100 range $(100=$ better QoL). A total HDQoL-C score has also been suggested [18] and is computed as the overall sum, transformed into a $0-100$ range $(100=$ better QoL). In addition, a short-form of the HDQoL-C has been proposed [21]. The short-form consists of a 3item version of the SL scale, the full FL scale and a total score; short-form item scores are summed and transformed into a $0-100$ range $(100=$ better $\mathrm{QoL})$ for each suggested scale [22]. In this study, short-form scores were computed from the full HDQoL-C.

The ACQLI was developed for AD carers [12, 19, 20] based on the needs-based QoL model [23, 24] and its 30 items (Table 1) were derived from in-depth interviews with $40 \mathrm{AD}$ carers; content validity was assessed by AD carers from five countries [12, 20]. Items are assumed to be unidimensional (i.e., represent a single variable), and are scored $0 / 1$ (not true/true) before summation into a total score with a $0-30$ range. To ease comparisons between scales in this study, ACQLI scores were transformed to range between 0-100 $(100=$ worse QoL $)$.

\section{Analyses}

Data were analysed using IBM SPSS 20 and ScoreRel CI [25]. Data completeness was studied by calculating the percentage of missing data for items and total scores, which should be low $(<10 \%)$ to be considered acceptable among responders $[26,27]$. In 
Table 1

Contents of the HDQoL-C and ACQLI

\begin{tabular}{|c|c|c|c|}
\hline \multicolumn{2}{|r|}{ HDQoL-C scales and items } & \multicolumn{2}{|r|}{ ACQLI items } \\
\hline No. & Content (abridged) & No. & Content (abridged) \\
\hline \multicolumn{2}{|c|}{ Practical aspects of Caregiving $(P C)$} & 1 & Never time to sit down \\
\hline 1 & Restricted by routine & 2 & Often irritable \\
\hline 2 & Appropriate social service help & 3 & Difficult organising shopping \\
\hline 3 & Access to HD professionals & 4 & Emotionally draining \\
\hline 4 & Support by health care professionals & 5 & No conversation \\
\hline 5 & Impact of genetic implications & 6 & Little freedom \\
\hline 6 & Care facilities access & 7 & Situation wears me down \\
\hline 7 & Practical support & 8 & Unable share feelings \\
\hline 8 & Conflict of interest & 9 & Constant pressure \\
\hline 9 & Sleep well & 10 & Lonely \\
\hline \multicolumn{2}{|c|}{ Satisfaction with Life (SL) } & 11 & Physically drained \\
\hline 1 & Health & 12 & Want to stop and cry \\
\hline 2 & Achievements in life & 13 & Living hell \\
\hline 3 & Family/friends relationships & 14 & Awake most of the night \\
\hline 4 & Feel safe & 15 & Low \\
\hline $5^{\mathrm{a}}$ & Feeling part of community & 16 & Takes all energy \\
\hline $6^{\mathrm{a}}$ & Happiness & 17 & Nobody calls \\
\hline 7 & Treatment of HD person & 18 & Always on my mind \\
\hline $8^{\mathrm{a}}$ & Overall QoL & 19 & Push to go on \\
\hline \multicolumn{2}{|c|}{ Feelings about Living with $H D(F L)$} & 20 & Losing independence \\
\hline $1^{\mathrm{a}}$ & Guilty & 21 & Little time to look after self \\
\hline $2^{\mathrm{a}}$ & Financially disadvantaged & 22 & No-one to turn to \\
\hline $3^{\mathrm{a}}$ & Isolated & 23 & Household jobs hard \\
\hline $4^{\mathrm{a}}$ & Hope & 24 & Neglecting appearance \\
\hline $5^{\mathrm{a}}$ & Exhausted & 25 & Tired all the time \\
\hline $6^{\mathrm{a}}$ & Supported & 26 & Illness ruins life \\
\hline $7^{\mathrm{a}}$ & Sad/depressed & 27 & Want to run away \\
\hline $8^{\mathrm{a}}$ & Stressed & 28 & Being with a stranger \\
\hline $9^{a}$ & Worried genetics & 29 & Situation makes me ill \\
\hline $10^{\mathrm{a}}$ & My needs not important & 30 & Feel guilty if not there \\
\hline $11^{\mathrm{a}}$ & Comforted - future HD cure & & \\
\hline $12^{\mathrm{a}}$ & Positive HD impact & & \\
\hline $13^{\mathrm{a}}$ & Comforted by beliefs & & \\
\hline $14^{\mathrm{a}}$ & Can cope & & \\
\hline $15^{\mathrm{a}}$ & HD made me stronger & & \\
\hline $16^{\mathrm{a}}$ & Duty of care forced on me & & \\
\hline $17^{\mathrm{a}}$ & Don't know who I am & & \\
\hline
\end{tabular}

addition, the time taken to complete each questionnaire was noted for the eight respondents who completed the questionnaires in the presence of the investigator, and was used as an indicator of questionnaire user friendliness. Up to about 10 minutes has been suggested as acceptable questionnaire completion time [28].

Scaling assumptions according to traditional psychometric criteria were then explored by examining the legitimacy of summing item scores into total scores without weighting or standardisation [27, 29]. That is, we examined whether item means and SDs were roughly similar across items within scales, and if each item contributed substantially to the total score (which is considered satisfied if corrected item-total correlations exceed 0.3) [29]. With dichotomously scored items, such as with the ACQLI, wider ranges of mean item scores have been considered acceptable [29]. Furthermore, we tested if data support the assumption that items within each proposed scale represent a single variable (indicated by corrected item-total correlations exceeding 0.3-0.4) [27, 29]. For the multidimensional HDQoL-C, we then examined whether the proposed grouping of items into scales was supported. To support this, each item's corrected item-total correlation with its proposed scale should exceed its correlation with any of the other HDQoL-C scales (referred to as scaling success) $[26,27]$.

Targeting was analyzed by examining how well the distribution of scores accorded with the range of QoL in the sample [29]. This was evaluated by studying score 
Table 2

Descriptive and psychometric data for the HDQoL-C, HDQoL-C short-form, and ACQLI among carers of people with HD

\begin{tabular}{|c|c|c|c|c|c|c|c|}
\hline & \multicolumn{4}{|c|}{ HDQoL-C } & \multicolumn{2}{|c|}{ HDQoL-C short-form } & \multirow[b]{2}{*}{ ACQLI } \\
\hline & PC & SL & $\mathrm{FL}^{\mathrm{a}}$ & Total & SL & Total & \\
\hline \multicolumn{8}{|l|}{ Data completeness } \\
\hline Missing item responses $(\min -\max \%)^{\mathrm{c}}$ & $0-11.5$ & $0-3.3$ & $0-1.6$ & $0-11.5$ & $0-1.6$ & $0-1.6$ & $0-3.3$ \\
\hline Computable scale scores $(\%)$ & 80.3 & 95.1 & 96.7 & 75.4 & 98.4 & 95.1 & 96.7 \\
\hline \multicolumn{8}{|l|}{ Scaling assumptions } \\
\hline Item score mean $(\min -\max )^{\mathrm{d}}$ & $3.9-7.3$ & $2.6-4.6$ & $3.0-7.9$ & $2.6-7.9$ & $3.8-4.6$ & $3.0-7.9$ & $0.13-0.75$ \\
\hline Item score SD (min-max) $)^{\mathrm{e}}$ & $3.2-3.9$ & $2.7-3.1$ & $2.8-3.8$ & $2.7-3.9$ & $2.8-3.1$ & $2.8-3.8$ & $0.38-0.50$ \\
\hline Corrected item-total correlation, & 0.30 & 0.71 & 0.44 & 0.48 & 0.84 & 0.5 & 0.60 \\
\hline mean $(\min -\max )^{\mathrm{f}}$ & $(0.15-0.49)$ & $(0.47-0.88)$ & $(0.08-0.75)$ & $(0.12-0.79)$ & $(0.77-0.88)$ & $(0.08-0.78)$ & $(0.38-0.78)$ \\
\hline Scaling success $(\%)^{\mathrm{g}}$ & 39 & 94 & $62^{\mathrm{b}}$ & NA & 100 & NA & NA \\
\hline \multicolumn{8}{|l|}{ Targeting } \\
\hline Mean (SD) score ${ }^{\mathrm{h}}$ & $56.5(17.2)$ & $37.4(22.3)$ & $50.6(17.4)$ & $49.6(16.8)$ & $43.1(27.4)$ & $49.5(18.2)$ & $42(29.5)$ \\
\hline Median (q1-q3) score ${ }^{h}$ & $59(43-70)$ & $36(17.5-54)$ & $51(37-52)$ & $51(36-63)$ & $47(17-62)$ & $51(33.5-62.5)$ & $43(13-63)$ \\
\hline Min-max score ${ }^{i}$ & $13.3-93.3$ & $0-87.5$ & $14.7-93.5$ & $19.2-91.5$ & $0-100$ & $15-93.5$ & $0-100$ \\
\hline Floor/ceiling effects $(\%)^{\mathrm{j}}$ & $0 / 0$ & $3.3 / 0$ & $0 / 0$ & $0 / 0$ & $6.6 / 1.6$ & $0 / 0$ & $9.8 \% / 1.6 \%$ \\
\hline Skewness ${ }^{\mathrm{k}}$ & 0.28 & 0.22 & 0.09 & 0.07 & 0.22 & 0.11 & 0.21 \\
\hline \multicolumn{8}{|l|}{ Reliability } \\
\hline Coefficient $\alpha(95 \% \mathrm{CI})^{1}$ & $\begin{array}{c}0.62 \\
(0.44-0.76)\end{array}$ & $\begin{array}{c}0.91 \\
(0.87-0.99)\end{array}$ & $\begin{array}{c}0.84 \\
(0.77-0.89)\end{array}$ & $\begin{array}{c}0.92 \\
(0.88-0.94)\end{array}$ & $\begin{array}{c}0.92 \\
(0.88-0.95)\end{array}$ & $\begin{array}{c}0.88 \\
(0.84-0.92)\end{array}$ & $\begin{array}{c}0.95 \\
(0.94-0.95)\end{array}$ \\
\hline $\begin{array}{l}\text { Coefficient } \alpha \text { when item } \\
\text { deleted }(\min -\max )^{\mathrm{m}}\end{array}$ & $0.54-0.64$ & $0.88-0.92$ & $0.81-0.85$ & $0.91-0.92$ & $0.86-0.94$ & $0.87-0.89$ & $0.94-0.95$ \\
\hline SEM & 10.66 & 6.69 & 6.96 & 4.75 & 7.75 & 6.3 & 6.49 \\
\hline 95\% CI (SEM) & \pm 20.89 & \pm 13.11 & \pm 13.64 & \pm 9.31 & \pm 15.19 & \pm 12.35 & \pm 12.72 \\
\hline
\end{tabular}

${ }^{\mathrm{a}}$ The HDQoL-C FL scale is also part of the HDQoL-C Short-Form. ${ }^{\mathrm{b}}$ Scaling success for the FL scale in the HDQoL-C Short-Form was $53 \%$.

${ }^{\mathrm{c}}$ Should be $<10 \%$. ${ }^{\mathrm{d}}$ Should be roughly similar across items within scales. ${ }^{\mathrm{e}}$ Should be roughly similar across items within scales. ${ }^{\mathrm{f}}$ Should be $>0.3$ to support summation of raw item scores, and $>0.3-0.4$ to support a single underlying variable. ${ }^{\mathrm{g}}$ Should be close to $100 \%$. ${ }^{\mathrm{h}}$ Should be close to scale midpoint. i Should span most of the scale's score range. ${ }^{j}$ Should be $<15-20 \%$. ${ }^{\text {K }}$ Should be between -1 and +1 . 'Should be $\geq 0.80$. ${ }^{\mathrm{m}}$ Should not increase. HDQoL-C, Huntington's Disease Quality of Life battery for Carers; ACQLI, Alzheimer's Carer's Quality of Life Instrument; PC, Practical aspects of Caregiving; SL, Satisfaction with Life; FL, Feelings about Living with HD; SD, standard deviation; q1-q3, 1st-3rd quartile (25th-75th percentile); CI, confidence interval; SEM, standard error of measurement; NA, not applicable.

distributions, skewness and floor and ceiling effects. A well-targeted scale should have an average score close to the scale midpoint and span most of its potential range, without excess skewness (preferable between -1 and +1$)$. Floor and ceiling effects refer to the proportions of participants that score minimum (floor) and maximum (ceiling) respectively. Up to 15-20\% floor/ceiling effects can be considered acceptable [29].

Reliability was assessed by coefficient alpha, for which coefficients above 0.8 are desirable [29]. The influence on coefficient alpha of deleting each item one at a time was also explored; an increased coefficient alpha following item deletion suggests problems with construct conceptualisation or multidimensionality [30]. In addition, the standard error of measurement $(\mathrm{SEM})$ was calculated $(\mathrm{SD} \times \sqrt{ } 1$-reliability $)$ as an estimator of score precision and was used to construct $95 \%$ confidence limits around scores [30].

To test if HDQoL-C scales appear to represent different aspects the correlations between them were compared with each scale's internal consistency (coef- ficient alpha). Scales are considered to represent distinct aspects if their internal consistencies are larger than their inter-correlations [27]. HDQoL-C and ACQLI scores were also correlated to assess to what extent these appear to represent the same variable.

\section{RESULTS}

Data completeness was generally good, but compromised for the PC scale (HDQoL-C), suggesting suboptimal acceptability among respondents (Table 2). Consequently, total PC scores could not be computed for about one fifth of the sample; HDQoL-C total scores could not be computed for about $25 \%$ of participants. Respondents needed an average of 27 and 5 minutes to complete the HDQoL-C and ACQLI, respectively, resulting in time-to-number of items ratios of 0.79 (HDQoL-C) and 0.17 (ACQLI).

Item mean scores were roughly similar for SL items but more dissimilar in the other HDQoL-C scales (Table 2). Although ACQLI item means covered a relatively wide range, this can be considered acceptable for 
dichotomously scored items (29). All item SDs were fairly similar within the respective scales (Table 2). Corrected item-total correlations exceeded 0.3 for the SL scales of the HDQoL-C and the ACQLI, whereas other HDQoL-C scales failed to meet this criterion (Table 2). Except for the SL scales (94\% and 100\% scaling success rates for the full and short forms, respectively), data did not support the grouping of items into the assumed HDQoL-C scales (Table 2). Collectively, these observations suggest that the SL scales (HDQoL-C) and the ACQLI represent single underlying variables, and support the legitimacy of their respective total scores.

Targeting was generally good as scores spanned the full (ACQLI) or almost the full (HDQoL-C) range of possible scale scores, median and mean scores approached scale midpoints (which in all instances was within one SD of observed averages), and skewness values were $<1$ (Table 2). Floor and ceiling effects were $<10 \%$ (Table 2).

Reliability was acceptable for all scores but the HDQoL-C PC scale (Table 2). Coefficient alpha increased slightly when items were deleted for all HDQoL-C scales but not for the ACQLI, suggesting some problems at the item level of the HDQoL-C. SEM values and associated $95 \%$ CIs were largest for the HDQoL-C PC scale and smallest for the HDQoL-C total scores and the ACQLI, indicating relatively better measurement precision of the latter.

Table 3 shows the correlations between scales in relation to their respective alpha coefficients. These data indicate that the PC scale does not appear to represent an aspect distinct from other HDQoL-C scales, whereas the SL and FL scales do (albeit marginally for the FL, taking the $95 \% \mathrm{CI}$ of its coefficient alpha

Table 3

Inter-scale correlations

\begin{tabular}{lccccc}
\hline & & \multicolumn{3}{c}{ HDQoL-C } \\
\cline { 3 - 5 } & ACQLI & PC & SL & FL \\
\hline ACQLI & $(0.95)^{\mathrm{a}}$ & & & \\
HDQoL-C PC & 0.53 & $(0.62)$ & \\
HDQoL-C SL & 0.80 & 0.55 & $(0.91)$ \\
HDQoL-C FL & 0.75 & 0.74 & 0.75 & $(0.84)$ \\
& & & & HDQoL-C (Short-Form) \\
\cline { 3 - 5 } & & & SL & FL \\
HDQoL-C SL (Short-Form) & 0.74 & & $(0.92)$ & \\
HDQoL-C FL (Short-Form) & 0.75 & & 0.74 & $(0.84)$ \\
\hline
\end{tabular}

${ }^{a}$ Internal consistency reliability (coefficient alpha) in parentheses. HDQoL-C, Huntington's Disease Quality of Life battery for Carers; ACQLI, Alzheimer's Carer's Quality of Life Instrument; PC, Practical aspects of Caregiving; SL, Satisfaction with Life; FL, Feelings about Living with HD. into account; see Table 2). The ACQLI correlated stronger with the SL and FL scales than with the PC scale, and its coefficient alpha exceeded its correlations with all HDQoL-C scales (Table 3). This suggests that the ACQLI represents a different construct from the HDQoL-C.

\section{DISCUSSION}

This study presents a head-to-head psychometric comparison of two carer QoL questionnaires, the HDQoL-C and the ACQLI among HD carers. To the best of our knowledge, this is the first independent comparative assessment of the psychometric properties of these scales. Our findings are in general agreement with earlier data on these scales [10, 12, 19-21], but also extend previous observations by considering aspects not reported before. Results suggest that two of the three proposed HDQoL-C scales, and its total scores fail to meet traditional psychometric criteria; the third scale (SL) does not outperform the AD targeted ACQLI. These observations imply that carer impact is not disease specific across neurodegenerative disorders and support the feasibility of a common neurodegenerative carer QoL questionnaire.

Data completeness supported the acceptability of both the HDQoL-C and ACQLI, except for the HDQoL-C PC scale (and consequently the HDQoL$\mathrm{C}$ total score), challenging its suitability among HD carers. In addition, the user friendliness of the HDQoL$\mathrm{C}$ may be questioned as responders needed $>4$ times longer (correcting for different numbers of items) to complete this questionnaire than the ACQLI. These findings are in accordance with previous observations. Completion time in the original HDQoL-C study was 21 minutes [10], and AD and PD carers have needed an average of 9.7 and 5.4 minutes to complete the ACQLI [9]. Although e.g. item wording may play a role, one reason for this discrepancy could be the use of 11grade (HDQoL-C) compared to dichotomous response categories (ACQLI).

Our observations provide support for the legitimacy of summing item raw scores to total scores for the ACQLI and the SL scales of the HDQoL-C, but not for other proposed HDQoL-C scales. Although it therefore may be argued that this disqualifies the PC, FL and HDQoL-C total scores as valid scales, additional psychometric properties of the full questionnaire were addressed to explore its relative qualities. Indeed, we found that targeting was generally good for all scales. Similarly, with the exception of the PC scale, relia- 
bility standards were met and score precisions were reasonable. These observations are in general accordance with those in the original HDQoL-C and ACQLI studies $[10,12,20,21]$, except for the PC scale (coefficient alpha, 0.80 [10]), and provide tentative support for these scales' abilities to reflect changes and differences among HD carers.

Although psychometrically fundamental, reliability does not tell what is being measured. This is a matter of validity, to which dimensionality is central in that items in a scale should represent a single variable to allow for valid summation of item responses and score interpretation [30]. In this respect, our observations support the assumption that ACQLI items represent a common latent variable [12, 20, 24]. In contrast, except for the full and short-form SL scales, data do not support the unidimensionality of HDQoL-C scales or the suggested grouping of HDQoL-C items into scales, as demonstrated by inadequate corrected item-total correlations and poor scaling success rates. Further indications of dimensionality problems were found when studying the effects on coefficient alpha when items were deleted and correlations between HDQoL-C scales relative to their respective alpha coefficients. Indeed, although unacknowledged, all HDQoL-C scales exhibited signs of multidimensionality also in the original HDQoL-C evaluations, as suggested by principal component analyses $[10,21]$. The conceptual and theoretical underpinnings of the HDQoL-C, or its operationalization therefore appear questionable.

The exact reason for the failure of the PC, FL and total HDQoL-C scores to meet basic scaling assumptions, and the ambiguity as to what they represent cannot be determined. However, three things are interesting to note. First, available documentation on the development of the HDQoL-C fails to provide an explicit definition or theoretical model for its target variables [10]. Although it was based on an existing generic instrument comprising seven theoretically derived domains [31], this was not preserved in the resulting HDQoL-C [10]. Second, despite the intention to reflect subjective QoL, content validity of the HDQoL-C was not informed by carers but by QoL and HD experts [10]. Third, several items appear to be causal rather than indicator items [32]. Consequently, interpretability of psychometric methods such as those used here and previously $[10,21]$ may be compromised and the validity of the HDQoL-C as a latent variable measurement instrument is challenged [32]. This indicates that the HDQoL-C might be regarded primarily as an assessment tool or checklist, where item responses should be interpreted on their own rather than as latent variable indicators for measurement. These concerns are in contrast to observations on the ACQLI, for which the legitimacy of summing item scores into a total score that represents a single latent variable was supported. In contrast to the HDQoL-C, the ACQLI was developed based on a theoretical QoL model and items were derived directly from carers, as was assessment of content validity $[12,20,23,24]$. Whereas it cannot be "proven" that ACQLI scores are valid QoL measures, and more sophisticated psychometric analyses are needed, these aspects represent major advantages that may explain observed discrepancies between the HDQoL-C and ACQLI.

We observed relatively strong correlations between ACQLI and SL scores, suggesting that they represent similar constructs. Indeed, life satisfaction has been proposed as an appropriate operationalization of QoL [33]. The observed relationship is therefore not surprising. However, caution is urged against equating life satisfaction with QoL [34]. Indeed, our observations suggest that the SL and ACQLI scales represent different constructs and their correlation implies that about one third of the variation in ACQLI scores cannot be explained by variations in SL scores (and vice verse). With the short-form SL scale, this relationship was even weaker (about $45 \%$ unexplained variance).

We recognize that the current sample size is relatively small and that it is unclear how representative it is for the HD carer population at large. However, since HD is a rare disorder the sample is likely to be more representative than otherwise would have been the case. Furthermore, samples smaller than this have been found sufficient in producing stable reliability and validity indices according to traditional psychometric methods such as those used here [35]. However, further evaluations based on larger samples are warranted. Such studies should also consider using modern test theory methodologies to allow for firmer psychometric testing [29].

Despite these limitations, this study allows for some preliminary but important conclusions and recommendations. First, we were unable to obtain support for the validity or reliability of two out of three HDQoL-C scales (PC and FL) as well as its total scores. This is particularly concerning since the HDQoL-C primarily has been suggested as an outcome measure [10, 21]. In contrast, we found tentative support for the psychometric properties of the ACQLI and the full and short-form HDQoL-C SL scales. Interestingly, the SL scales failed to display advantages over the ACQLI despite being devised specifically for HD carers. However, scale 
selection should be driven by the desired target variable and study objectives $[17,36]$. Therefore, the ACQLI appears useful for measuring carer QoL, whereas the HDQoL-C SL scales are preferable if life satisfaction is the variable of interest. Of the two SL scale versions, the full version appears superior due to better measurement precision. However, the relative merits of this scale versus more established life satisfaction questionnaires are unknown. Importantly, our findings suggest that any revisions of the HDQoL-C should reconsider the feasibility of the 11-grade response format, attend to its conceptual underpinnings, define its target variable(s), and review item selection to improve its representation of the intended variable(s), which are necessary first steps for successful measurement. Finally, our observations regarding the AQQLI have two related implications. First, they provide tentative support for the idea of a common cross-diagnostic carer impact of neurodegenerative disorders $[2,8,9]$. Secondly, and in accordance with previous observations among PD and AD carers [9], the AQQLI appears a promising candidate tool for further work towards such cross-diagnostic QoL assessments.

\section{ACKNOWLEDGMENTS}

The authors wish to thank all participating respondents for their cooperation, and Dr. Roger Barker for assistance with participant recruitment. The study was supported by the Swedish Research Council, and accomplished within the Basal Ganglia Disorders Linnaeus Consortium (BAGADILICO) research group at Lund University, Lund, Sweden.

\section{CONFLICTS OF INTEREST}

No authors have any conflict of interest to report.

\section{AUTHOR ROLES}

P.H. conceived and organized the research project; wrote the first manuscript draft; designed, executed and interpreted the statistical analyses. S.S. conceived, organized and executed the research project, executed and interpreted the statistical analyses. Both authors reviewed, critiqued and approved the final manuscript.

\section{REFERENCES}

[1] Aguera-Ortiz L, Frank-Garcia A, Gil P, Moreno A. Clinical progression of moderate-to-severe Alzheimer's disease and caregiver burden: A 12-month multicenter prospective observational study. Int Psychogeriatr. 2010;22(8):1265-79.

[2] Brodaty H. Meaning and measurement of caregiver outcomes. Int Psychogeriatr. 2007;19(3):363-81.

[3] Peters M, Fitzpatrick R, Doll H, Playford D, Jenkinson C. Does self-reported well-being of patients with Parkinson's disease influence caregiver strain and quality of life? Parkinsonism Relat Disord. 2011;17(5):348-52.

[4] Roscoe LA, Corsentino E, Watkins S, McCall M, SanchezRamos J. Well-being of family caregivers of persons with late-stage Huntington's disease: Lessons in stress and coping. Health communication. 2009;24(3):239-48.

[5] Smith S. The neuro care manual: A guide to neurology for nurses and family carers. New Delhi: B. Jain Publishers (P) Ltd, 2011.

[6] Jankovic J, Tolosa E. Parkinson's disease and movement disorders, Fourth edition. Philadelphia: Lippincott Williams and Wilkins, 2002

[7] Thommessen B, Aarsland D, Braekhus A, Oksengaard AR, Engedal K, Laake K. The psychosocial burden on spouses of the elderly with stroke: Dementia and Parkinson's disease. Int J Geriatr Psychiatry. 2002;17(1):78-84.

[8] Habermann B, Davis LL. Caring for family with Alzheimer's disease and Parkinson's disease: Needs, challenges and satisfaction. J Gerontol Nurs. 2005;31(6):49-54.

[9] Hagell P, Hariz GM. Assessment of the Alzheimer Carer Quality of Life Instrument (ACQLI) among Swedish Alzheimer's and Parkinson's disease carers. Parkinsonism Relat Disord. 2009; 15(Suppl 2): S138.

[10] Aubeeluck A, Buchanan H. The Huntington's disease quality of life battery for carers: Reliability and validity. Clin Genet. 2007;71(5):434-45.

[11] Pickett T, Jr., Altmaier E, Paulsen JS. Caregiver burden in Huntington's disease. Rehabil Psychol. 2007;52(3):311-8.

[12] Doward LC. The development of the Alzheimer's carers' quality of life instrument (ACQLI). Qual Life Res. 1997;6(Suppl 7-8):639.

[13] Jenkinson C, Dummett S, Kelly L, Peters M, Dawson J, Morley D. et al. The development and validation of a quality of life measure for the carers of people with Parkinson's disease (the PDQ-Carer). Parkinsonism Relat Disord. 2012;18(5):483-7.

[14] Smith SC, Lamping DL, Banerjee S, Harwood RH, Foley B, Smith P. et al. Development of a new measure of health-related quality of life for people with dementia: DEMQOL. Psychol Med. 2007;37(5):737-46.

[15] Vickrey BG, Hays RD, Maines ML, Vassar SD, Fitten J, Strickland T. Development and preliminary evaluation of a quality of life measure targeted at dementia caregivers. Health Qual Life Outcomes. 2009;7:56

[16] Doward LC, Meads DM, Thorsen H. Requirements for quality of life instruments in clinical research. Value Health. 2004;7(Suppl 1):S13-6.

[17] Hobart J. Rating scales for neurologists. J Neurol Neurosurg Psychiatry. 2003;74(Suppl 4):iv22-iv6.

[18] Aubeeluck A, Buchanan H. Huntington's Disease Quality of Life Battery for Carers (HDQoL-C) - Validated for use with family Carers of Persons with Huntington's Disease [user guide]. Nottingham, UK: University of Nottingham, 2007.

[19] Brod M, Doward LC, McKenna S, Whalley D. The measurement of caregiver QoL and US adaptation of the Alzheimer's Caregivers Quality of Life Instrument (ACQLI). Qual Life Res. 2000;9(Suppl):26.

[20] Doward L. The ACQLI: An instrument to measure the Quality of Life of Alzheimer's Carers - User's manual. Manchester: Galen Research, 1997. 
[21] Aubeeluck A, Dorey J, Squitieri F, Clay E, Stupple EJ, De Nicola A. et al. Further evidence of reliability and validity of the Huntington's disease quality of life battery for carers: Italian and French translations. Qual Life Res. 2013;22(5): 1093-8.

[22] Aubeeluck A, Dorey JMT, Buchanan H. Huntington's Disease Quality of Life Battery for Carers - Short Form (HDQoLCSF) - Validated for use with Family Carers of Persons with Huntington's Disease. User guide for English and other translations. Nottingham, UK: University of Nottingham, 2009.

[23] Hunt SM, McKenna SP. The QLDS: A scale for the measurement of quality of life in depression. Health Policy. 1992;22(3):307-19.

[24] McKenna SP, Doward LC. The needs-based approach to quality of life assessment. Value Health. 2004;7(Suppl 1):S1-S3.

[25] Barnette JJ, ScoreRel. CI: An Excel program for computing confidence intervals for commonly used score reliability coefficients. Educ Psychol Meas. 2005;65:980-3.

[26] Saris-Baglama RN, Dewey CJ, Chisholm GB, Kosinski M, Bjorner JB, Ware JE, Jr. SF health outcomes ${ }^{\mathrm{TM}}$ scoring software user's guide. Lincoln: QualityMetric, Inc, 2004

[27] Ware JE, Jr., Gandek B. Methods for testing data quality, scaling assumptions, and reliability: The IQOLA Project approach. International Quality of Life Assessment. J Clin Epidemiol. 1998;51(11):945-52.

[28] McKenna SP. Measuring quality of life in schizophrenia. Eur Psychiatry. 1997;12(Suppl 3):267s-74s.
[29] Hobart J, Cano S. Improving the evaluation of therapeutic interventions in multiple sclerosis: The role of new psychometric methods. Health Technol Assess. 2009;13(12):iii, ix-X, 1-177.

[30] Nunnally JC, Bernstein IH. Psychometric theory. 3rd ed. New York: McGraw-Hill, Inc, 1994.

[31] Commins RA. The Comprehensive Quality of Life scale Adult, Fifth edition (ComQoL-A5) Manual. Melbourne: Deakin University. 1997.

[32] Fayers PM, Hand DJ. Causal variables, indicator variables and measurement scales: An example from quality of life. J R Stat Soc Ser A Stat Soc. 2002;165(2):233-66.

[33] Moons P, Budts W, De Geest S. Critique on the conceptualisation of quality of life: A review and evaluation of different conceptual approaches. Int J Nurs Stud. 2006;43(7):891-901.

[34] Hagell P, Westergren A. The significance of importance: An evaluation of Ferrans and Powers' Quality of Life Index. Qual Life Res. 2006;15(5):867-76.

[35] Hobart JC, Cano SJ, Warner TT, Thompson AJ. What sample sizes for reliability and validity studies in neurology? J Neurol. 2012;259(12):2681-94.

[36] Food and Drug Administration. Patient-Reported Outcome measures: Use in Medicinal Product Development to Support Labelling Claims. Washington DC, 2009. 